\title{
Photoinduced modifications by fluoroquinolone drugs in bovine serum albumin (BSA) and ribonuclease A (RNAse) as model proteins
}

\author{
Francesco Dall'Acqua ${ }^{a *}$, Giampietro Viola ${ }^{a}$, Daniela Vedaldi ${ }^{a}$, Gian Gaetano Aloisi ${ }^{\mathbf{b}}$, \\ Fausto Elisei $^{b}$, Loredana Latterini ${ }^{b}$, and Rosita Passeri ${ }^{b}$ \\ ${ }^{a}$ Department of Pharmaceutical Sciences, University of Padova, via Marzolo 5, 35131-Padova, \\ Italy \\ ${ }^{b}$ Department of Chemistry and CEMIN (Centro di Eccellenza sui Materiali Innovativi \\ Nanostrutturati), University of Perugia via Elce di Sotto 8, 06123-Perugia, Italy \\ E-mail: Francesco.dallacqua@unipd.it
}

Dedicated to Professor Waldemar Adam on the occasion of his $\mathbf{7 0}^{\text {th }}$ birthday

\begin{abstract}
Photosensitized protein oxidation by drugs, with the consequent modification of their structure is thought to be responsible for the occurrence of phototoxic phenomena such as photoallergy and loss of biological functions. In this paper we have investigated in detail the interaction of four fluoroquinolones namely ciprofloxacin, lomefloxacin, norfloxacin and ofloxacin with two proteins such as Bovine Serum Albumin (BSA) and Ribonuclease A (RNAse A) chosen as models. The interactions between the four drugs and the proteins, were studied by absorption and emission spectroscopy. Photophysical experiments were carried out in aqueous solutions by stationary and time-resolved fluorimetry and by laser flash photolysis, in the absence and in the presence of the proteins to obtain information on the various decay pathways of the excited states of the drugs and on transient species formed upon irradiation. In parallel we have investigated by a series of biochemical assays the photoinduced modifications exerted by the four drugs. The obtained results showed that the four drugs are able to photooxidize proteins with the formation of protein-protein cross-link. This effect was also confirmed in isolated erythrocyte membranes. Furthermore the effect of the fluoroquinolones was also evaluated on isolated aromatic aminoacids. In this context the four drugs are able to photodamage in particular tyrosine and histidine. These results are important in the light of the growing interest for the comprehension of the mechanism of phototoxicity induced by these antibacterial drugs.
\end{abstract}

\footnotetext{
* Prof. Francesco Dall'Acqua

Department of Pharmaceutical Sciences, University of Padova

Via Marzolo 5, 35131-Padova ITALY

Tel +3949 8275708, Fax: +39498275366

E-Mail: Francesco.dallacqua@unipd.it
} 
Keywords:. Fluoroquinolones, protein photodamages, fluorescence, protein binding, laser flash photolysis

\section{Introduction}

The fluoroquinolones are an important class of antimicrobials whose potential clinical usefulness continues to expand ${ }^{1}$. Quinolones are potent chemotherapeutic agents of first choice for the treatment of a broad range of bacterial infections, and their toxicity is low and comparable to that of other commonly used antimicrobial agents; thus quinolones can be considered to be relatively well-tolerated $^{2,3}$. However they occasionally produce side effects such as acute dermatitis which are usually confined to the areas of the skin exposed to sunlight. The phototoxic mechanism(s) as the cause of abnormal photosensitivity and its relationship to chemical structure remain unclarified.

Many studies on the phototoxic properties of these drugs have been reported in the last decades and the structure-side effects relationship of newly developed fluoroquinolones has been evaluated ${ }^{2}$. Primary acute and chronic lesions involving the photosensitized skin are erythema, oedema and pruritus, in more severe cases blistering and vesicles of the exposed parts ${ }^{4}$. These kinds of skin alterations may deteriorate into cancer ${ }^{5}$.

The action of these photosensitizers is dependent on the production of extremely reactive molecular species (reactive oxygen species, free radicals, photoproducts), able to modify the cell components, including lipids, proteins and nucleic acids, in combination with UVA; it has been suggested that the most phototoxic quinolones are those that induce the formation of reactive oxygen species (ROS), because these species cause severe tissue damages ${ }^{6-8}$. However, a correlation between the production of ROS and the phototoxic potency of fluoroquinolones cannot be drawn and other mechanisms, i.e. generation of highly reactive photodegradation products, seem to be involved ${ }^{9}$.

Concerning their mechanism of action it is known that they are able to photodamage DNA ${ }^{10}$, and to induce peroxidation of membrane lipids ${ }^{11}$. Instead scarce information is available regarding their capability to interact with proteins. Considering that proteins represent about the $60 \%$ of the dry weight of a cell these macromolecules may provide an important target in the phototoxic action of the fluoroquinolones. The purpose of this study is to evaluate the interactions and the chemical changes taking place upon irradiation in the two model proteins: Bovine Serum Albumin (BSA) and Ribonuclease A (RNAse A) in the presence of four fluoroquinolones (Figure 1), namely Ciprofloxacin (CPX), Lomefloxacin (LMX), norfloxacin (NOR) and Ofloxacin (OFX). 
<smiles>CCn1cc(C(=O)O)c(=O)c2cc(F)c(N3CCNC(C)C3)c(F)c21</smiles>

Lomefloxacin (LMX)<smiles>CCn1cc(C(=O)O)c(=O)c2cc(F)c(N3CCNCC3)cc21</smiles>

Norfloxacin (NOR)<smiles>CC1COc2c(N3CCN(C)CC3)c(F)cc3c(=O)c(C(=O)O)cn1c23</smiles>

Ofloxacin

(OFX)<smiles>O=C(O)c1cn(C2CC2)c2cc(N3CCNCC3)c(F)cc2c1=O</smiles>

Ciprofloxacin

$(\mathrm{CPX})$

Figure 1. Molecular structure of the fluoroquinolones investigated.

\section{Results and Discussion}

\section{Protein binding}

The binding interactions between the fluoquinolones under investigation and BSA or RNAse were investigated by use of steady state absorption and emission techniques. Figure 2 shows, as an example, the absorption spectra of CPX in PBS buffer $(\mathrm{pH}=7.2)$ in the presence of increasing concentrations of BSA (Figure2, Panel a) and RNAse (Figure2, panel b). Generally, after correction for dilution, an increase of the absorbance in the range 300-400 $\mathrm{nm}$ (where the drug transitions are located) occur with increasing the protein content in the sample solution. The effect appeared stronger with BSA taking into account that at protein concentrations higher than $2 \times 10^{-5} \mathrm{M}$ no further spectral changes were observed (saturation conditions) suggesting that all the drug is bound. This behaviour is consistent with the formation of one complex with a determined structure and stoichiometry which do not change upon increasing the protein concentration. The absorbance $v s$ protein concentration data were analyzed as Scatchard plot $^{12}$ which allowed the binding parameters for the interaction process to be obtained. For all the drugs under investigation, the analysis gave a binding constant $\left(\mathrm{K}_{\mathrm{a}}\right)$ value around $10^{5} \mathrm{M}^{-1}$ and the number of sites (n) per drug molecule involved in the interaction close to the unity. These binding parameters appear in good agreement with literature data reported for similar 
compounds $^{13,14}$. In the case of RNAse the saturation condition was not reached even for protein concentrations higher than $4 \times 10^{-4} \mathrm{M}$. This behaviour made more uncertain the determination of $\mathrm{K}_{\mathrm{a}}$ and values one order of magnitude lower $\left(10^{4} \mathrm{M}^{-1}\right)$ compared to those determined for BSA were estimated.

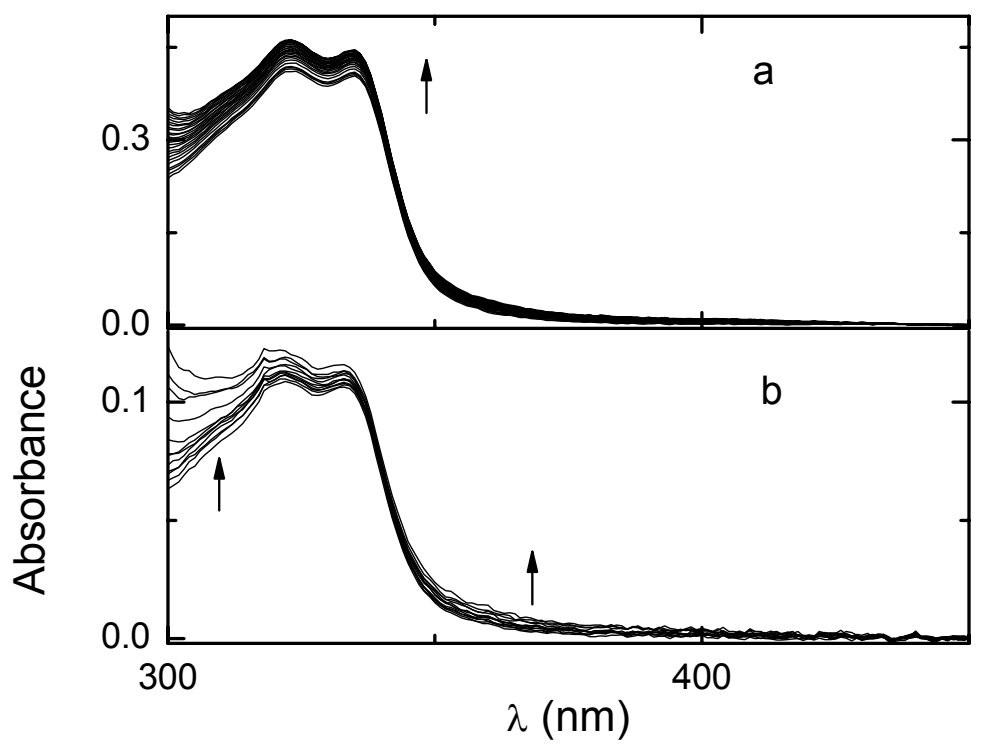

Figure 2. Absorption spectra of $\mathrm{CPX}$ in $\mathrm{PBS}$ buffer $\mathrm{pH}=7.2$ in the presence of increasing concentrations of (a) BSA $\left(0-1.31 \times 10^{-5} \mathrm{M}\right)$ and (b) RNAse $\left(0-2.7 \times 10^{-4} \mathrm{M}\right)$.

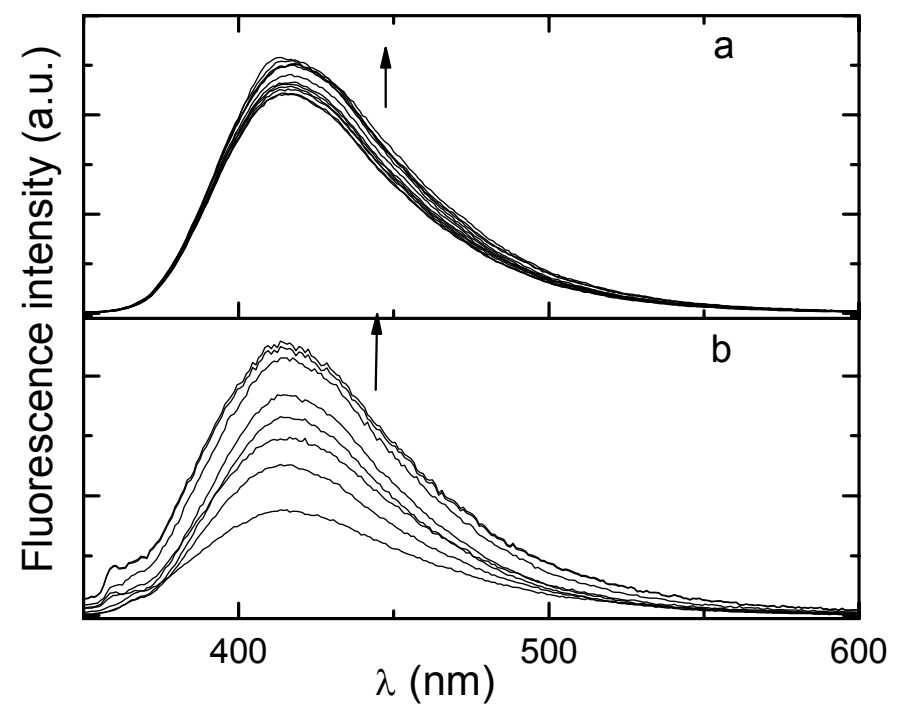

Figure 3. Fluorescence spectra of CPX (a) with increasing concentration of RNAse (0 $\left.2.7 \times 10^{-4} \mathrm{M}, \lambda_{\mathrm{exc}}=334 \mathrm{~nm}\right)$ and LMX (b) with increasing concentration of BSA $\left(0-1.7 \times 10^{-5} \mathrm{M}\right.$, $\left.\lambda_{\mathrm{exc}}=325 \mathrm{~nm}\right)$. 
The fluorescence titration spectra, corrected for the fraction of absorbed light for each experiment, showed an increase of the emission intensity upon increase of protein concentration (see Figure 3 as an example). The relative increase of emission intensity depended on the system; in particular for the LMX-BSA the fluorescence enhanced by a factor of three (Figure 3, panel b) while for the OFX-BSA the intensity increased 1.2 times (data not shown). These observations indicated that the drug-BSA complex has a higher fluorescence efficiency compared to the free drug, probably because the biological environment makes the other deactivation paths (competing with fluorescence) of the drug less favourable. Similarly to what observed in the absorption spectra, the fluorescence intensity observed in the presence of increasing RNAse concentrations did not reach a plateau value.

\section{Laser flash photolysis}

Laser flash photolysis experiments were carried out in some systems to investigate the transient behaviour of the drugs when bound to the proteins. The results were compared with the data obtained from the free drug. Figures 4 and 5 report the time resolved spectra of NOR in PBS buffer and NOR-BSA system, respectively; the latter was recorded in the conditions where all the drug is in the complex form. The assignment of the signal to chemical species was performed on the basis of literature data ${ }^{15,16}$ and by analogy with the spectral feature observed for the free drug. Upon laser excitation the triplet state $\left(\lambda_{\max }=610 \mathrm{~nm}\right)$ is formed which decayed faster than that of the free molecule to produce radical species $\left(\lambda_{\max }=400,650 \mathrm{~nm}\right)$. The results indicated that the interactions with the protein would not alter the transient formation, but it appeared that the species decayed faster, especially the triplet state.

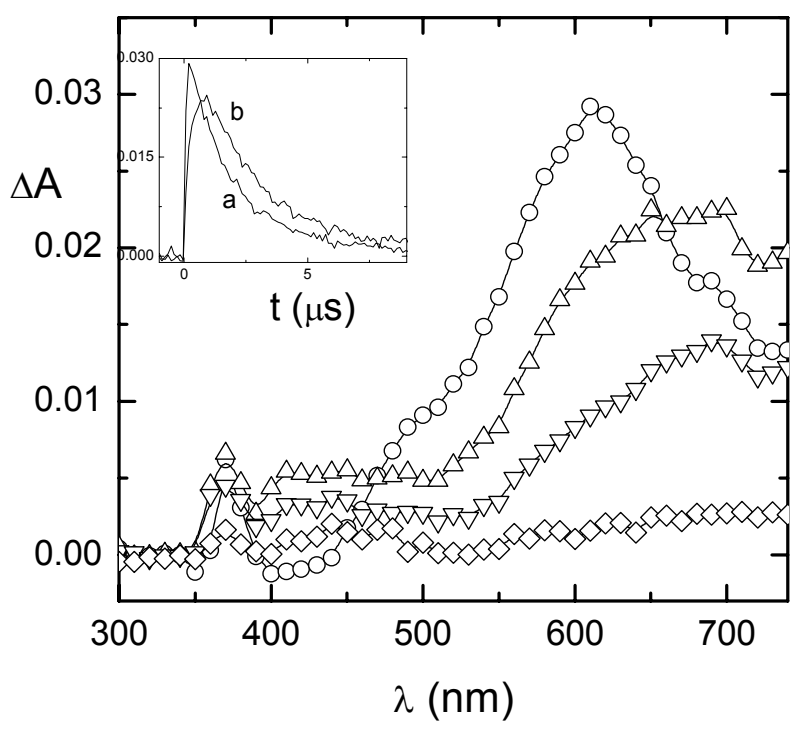

Figure 4. Time resolved spectra of NOR in PBS buffer $\mathrm{pH}=7.2$, recorded $0.2(\mathrm{o}), 1.0(\Delta)$, 2.5( $\nabla), 7.6(\diamond) \mu$ s after the laser pulse $\left(\lambda_{\text {exc }}=355 \mathrm{~nm}\right)$. Inset: Decay kinetics at 610 (a) and 700 (b) $\mathrm{nm}$. 


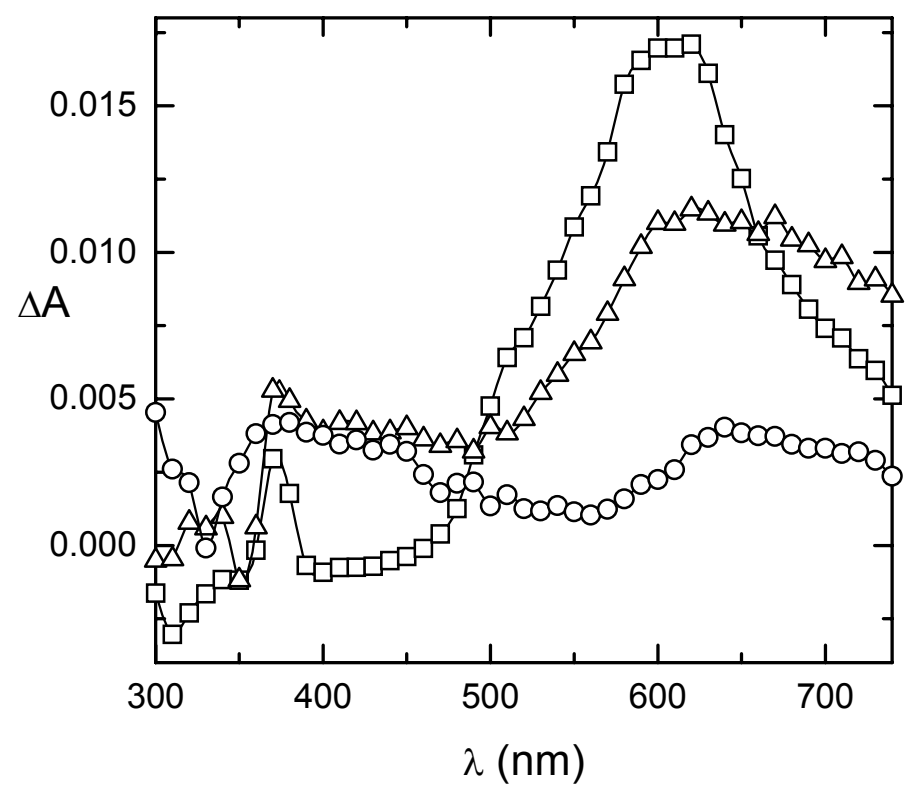

Figure 5. Time resolved spectra of NOR in PBS buffer $\mathrm{pH}=7.2$, in the presence of BSA $3.2 \times 10^{-4}$ $\mathrm{M}$ recorded $0.08(\square), 0.6(\Delta)$ and $5.0(\mathrm{o}) \mu$ s after the laser pulse $\left(\lambda_{\text {exc }}=355 \mathrm{~nm}\right)$.

\section{Protein photo-crosslink}

The photosensitization ability of the compounds towards the protein of cellular membranes, was estimated by measuring the photoinduced cross-linking in erytrocyte ghost proteins ${ }^{17}$. Lightinduced cross-linking of spectrin, a protein associated with the cytoplasmatic side of the RBC membrane, in the presence of the drugs was detected by the partial or total disappearance of the two spectrin bands (220.000 and 245.000 Daltons) on SDS-PAGE gel: cross-linked aggregates can not run inside the gel and remain at the top. Figure 6 show the picture of the SDS-PAGE gel of ghosts irradiated in the presence of CPX, as representative compound, in various conditions.

It can be observed under aerobic conditions that CPX causes an almost total disappearance of the two spectrin subunit bands whereas under nitrogen this effect is strongly reduced indicating the involvement of reactive oxygen species. The gel scans of erythrocyte spectrin, for all the four drugs investigated both in aerobic and anaerobic conditions, are also shown in Figure 6. OFX was the most powerful photosensitiser, followed by CPX, NOR and LMX. For all drugs the photoinduced cross linking is remarkably reduced in anaerobic conditions. 

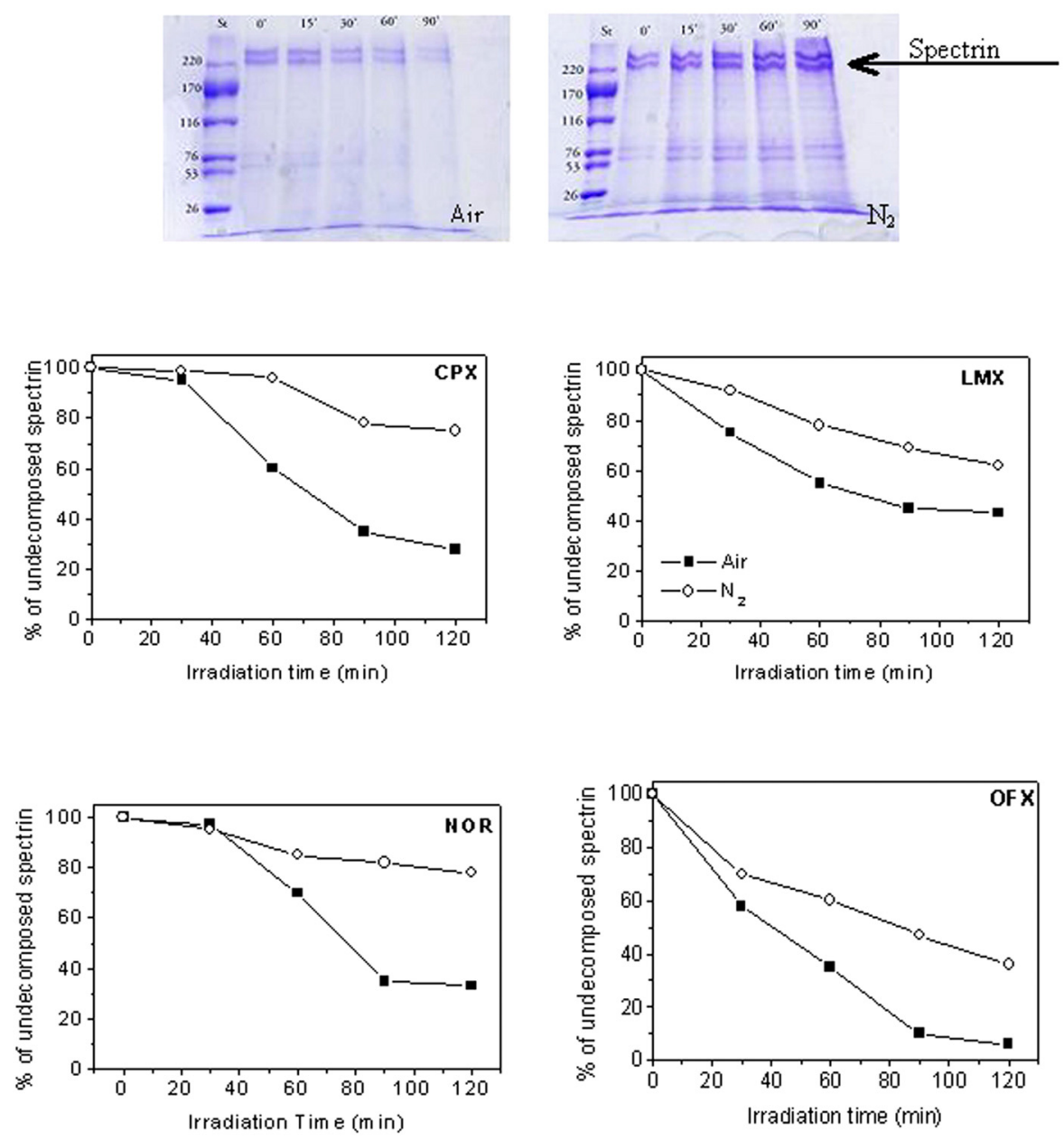

Figure 6. Electrophoretic pattern (upper panel) of the photoinduced cross-linking of spectrin in $\mathrm{RBC}$ ghosts irradiated at the indicates times in the presence of CPX at the concentration of 100 $\mu \mathrm{M}$ in aerobic (left) and anaerobic (rigth). Spectrin band is indicated by an arrow. St is a mixture of molecular weight standard, whose values are depicted in the left of the figure. Quantitation by gel densitometry of the relative abundance of spectrin after irradiation at various UV doses in the presence of fluoroquinolones (middle and lower panels).

The ability of the test compounds to photoinduce protein cross-linking was further investigated on RNAse A as protein model ${ }^{18}$. The obtained results depicted in Figure 7 indicate that OFX and LMX significantly induce, in a UVA-dose dependent way, the formation of a dimer of RNAseA. 


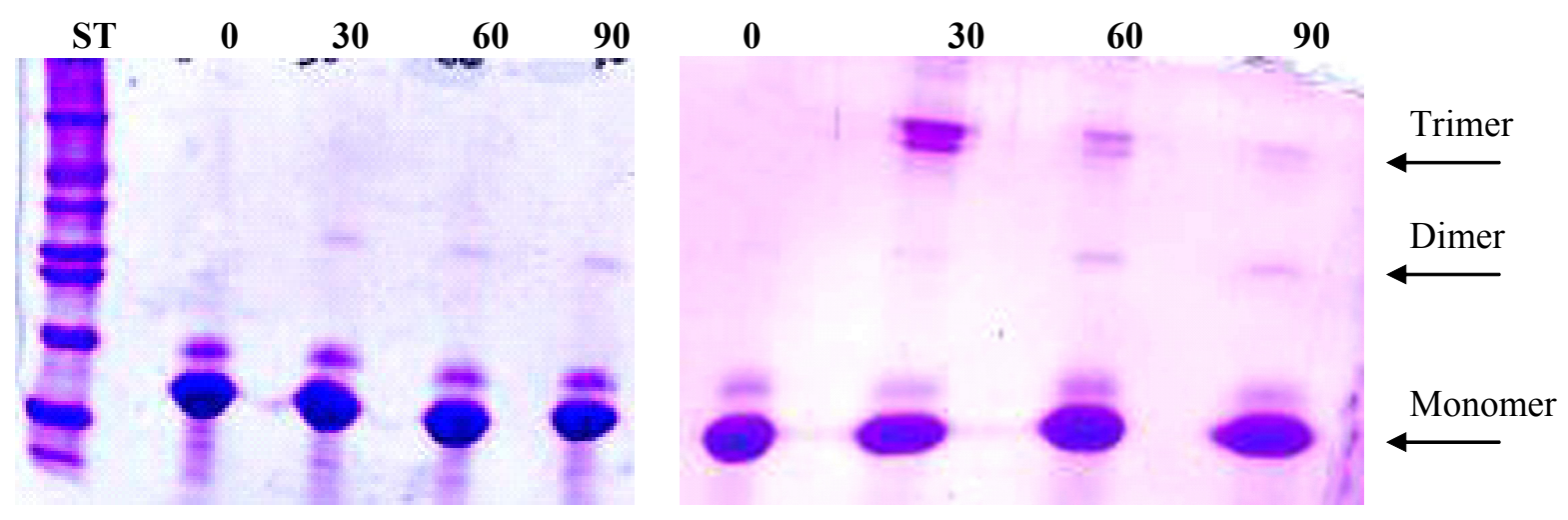

Figure 7. SDS-PAGE electrophoresis of RNAse irradiated in the presence of OFX (left panel) and LMX (right panel) at the concentration of $100 \mu \mathrm{M}$ and for the indicated times expressed in minutes.

\section{Protein photoxidation}

To investigate more deeply the phosensitizing properties of the three compounds toward proteins, solutions containing BSA or RNAse and the drugs in PBS were irradiated for various times. The degree of protein oxidation was monitored by the method of Levine ${ }^{19}$ which uses the reaction of 2,4-dinitrophenylhydrazine (DNPH) with the carbonyl groups of oxidized proteins. The results obtained (Figure 8) demonstrate that the four fluoroquinolones are able to increase the carbonyl content of both BSA and RNAse after exposure to UVA.
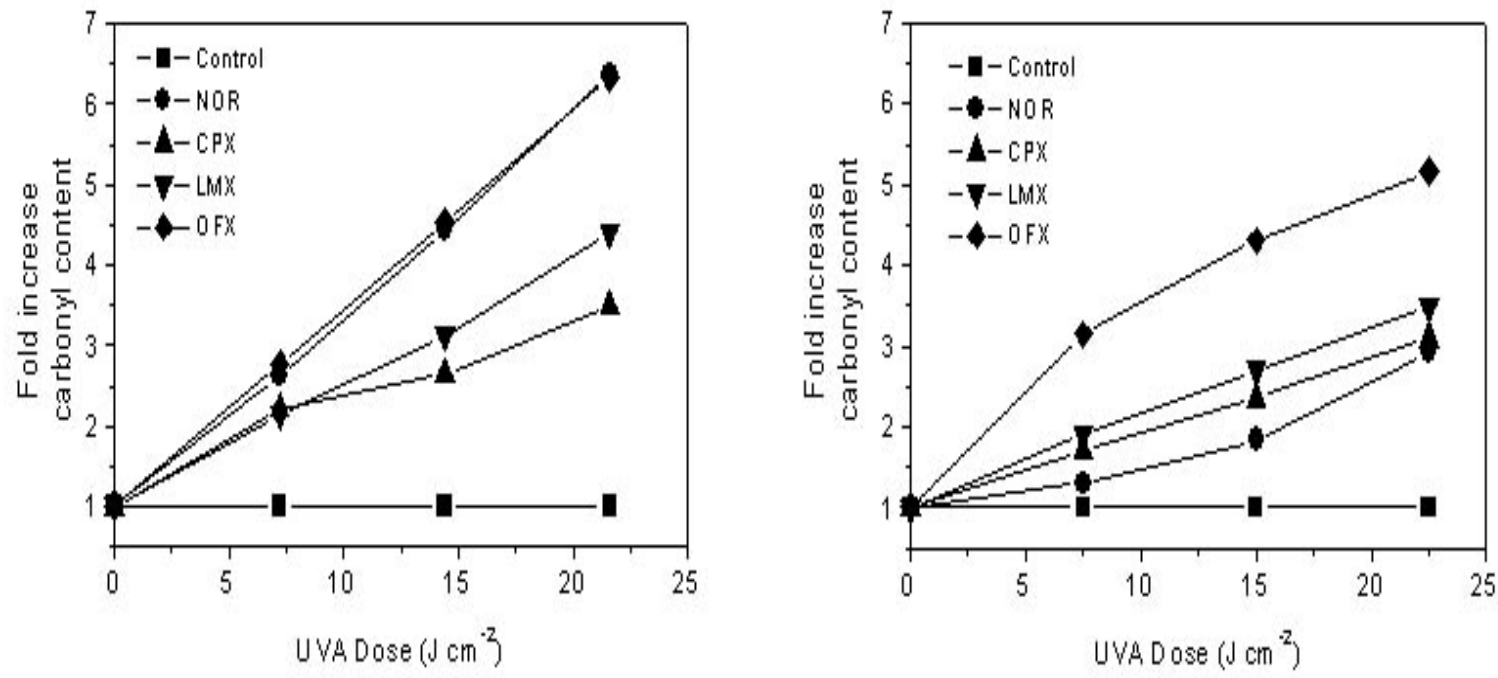

Figure 8. The increase in carbonyl content in BSA (left panel) and RNAse (right panel) after irradiation with fluoroquinolones at the concentration of $100 \mu \mathrm{M}$ and at the indicated UVA doses. 


\section{Modifications of Trp in BSA and Tyr in RNAse photoinduced by fluoroquinolones}

The Trp content was directly analyzed by monitoring the characteristic fluorescence of Trp residues in $\mathrm{BSA}^{20}$. As depicted in Figure 9, a rapid decrease of the fluorescence was observed under aerobic condition for CPX and NOR. No effects were observed when BSA was irradiated in the same conditions. When $\mathrm{N}_{2}$ was bubbled through the solution prior and during the irradiation in the presence of these two fluoroquinolones, no protection for the protein photodamage was observed suggesting that reactive oxygen species in this process are not involved. Furthermore, to corroborate this hypothesis, no significant differences were observed when the aqueous buffer was replaced by $\mathrm{D}_{2} \mathrm{O}$ which increase the lifetime of singlet oxygen, ${ }^{1} \mathrm{O}_{2}$ $\left({ }^{1} \Delta_{\mathrm{g}}\right)$. OFX in this assay was completely ineffective. An interesting behaviour was exhibited by LMX for which we observed an increase of the fluorescence. This effect could be due to the formation of a photoadduct between the protein and the drug as postulated by Tokura et al. ${ }^{21}$.
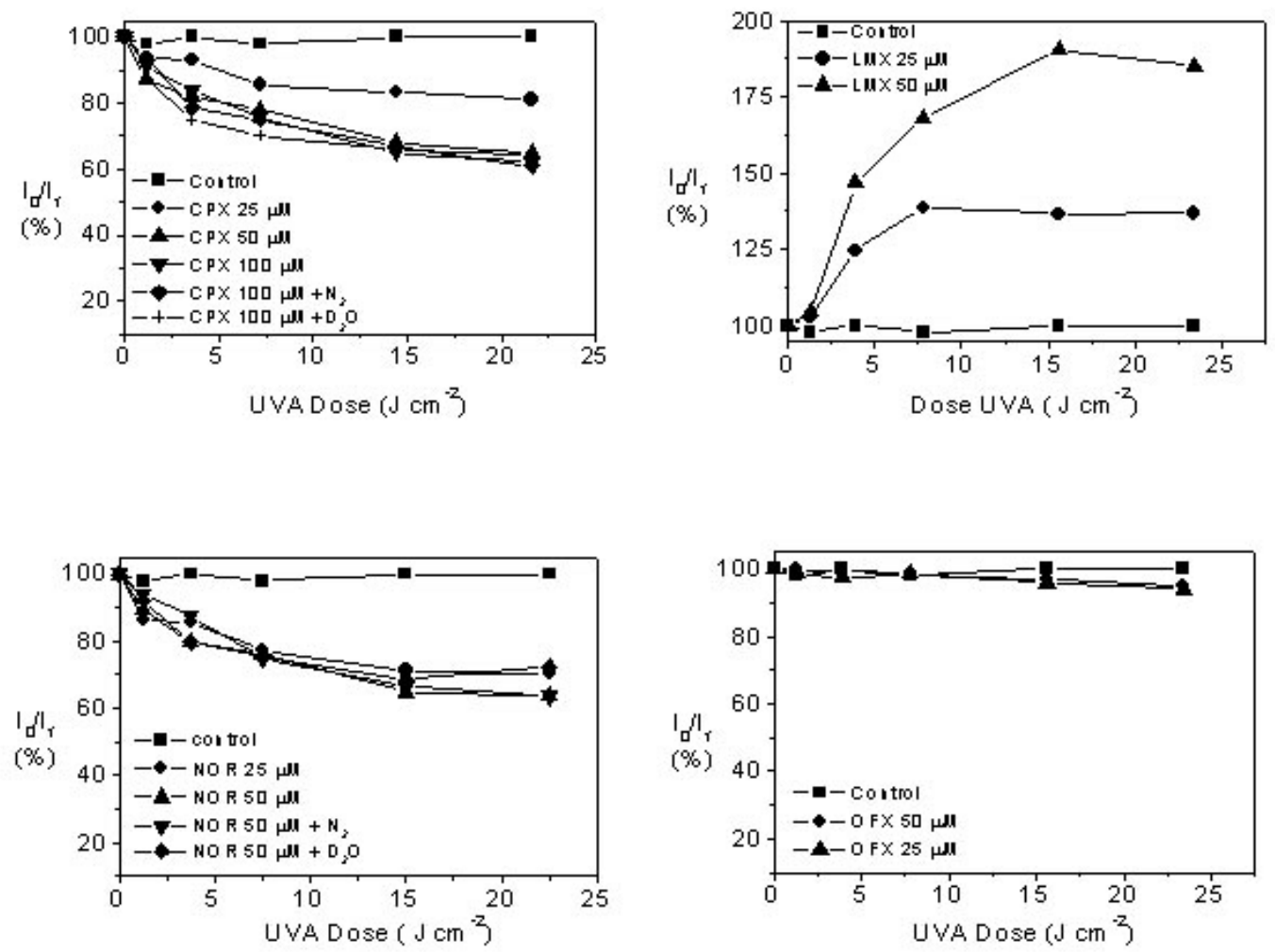

Figure 9. Photosensitising effects on BSA of fluoroquinolones after irradiation at the indicated concentrations, in different conditions.

Another aminoacid investigated in this study was Tyrosine (Tyr). We have used as a protein model RNAseA, which is a protein that is devoid of Trp but has Tyr residues in its sequence; as a consequence its fluorescence emission band, centred at about $350 \mathrm{~nm}$, is totally due to Tyr residues. As shown in Figure 10, the results obtained with RNAse are similar to those obtained 
with BSA suggesting a common effect of the fluoroquinolones both with Trp and Tyr. Interestingly also with this protein LMX exhibited the same behaviour observed with BSA. Further experiments are needed to clarify this point.
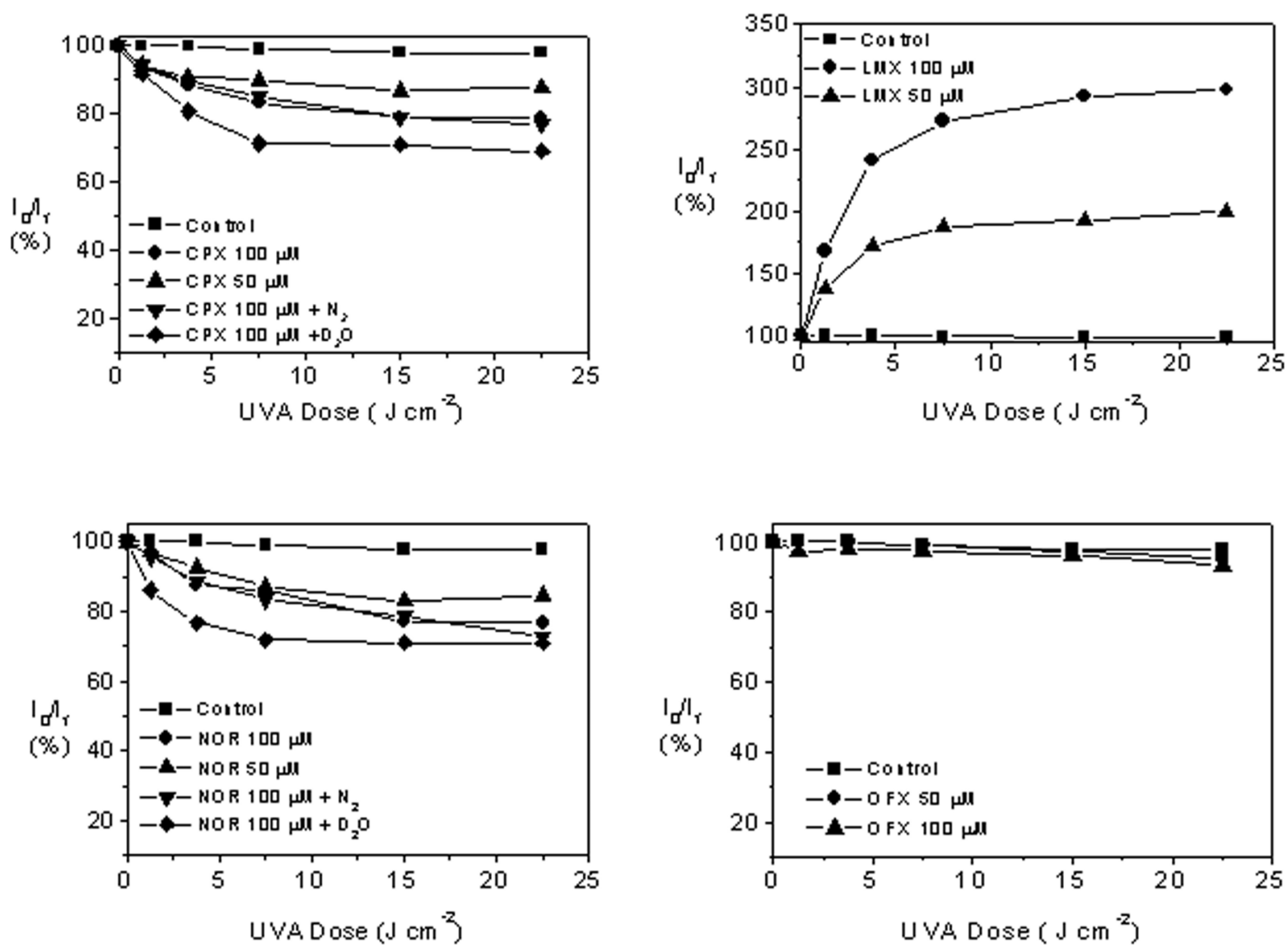

Figure 10. Photosensitising effects on RNAse of fluoroquinolones after irradiation at the indicated concentrations, in different conditions.

\section{Studies on isolated aminoacids}

To have a deeper insight into the mechanism of the protein photodamage induced by fluoroquinolones we performed the irradiation in the presence of pure isolated aminoacids such as Trp, Tyr and His. Unfortunately it was not possible to monitor Trp because, in the presence of the drugs, its fluorescence is significantly quenched. His and Tyr were monitored by colorimetric assay as described in the experimental section. 

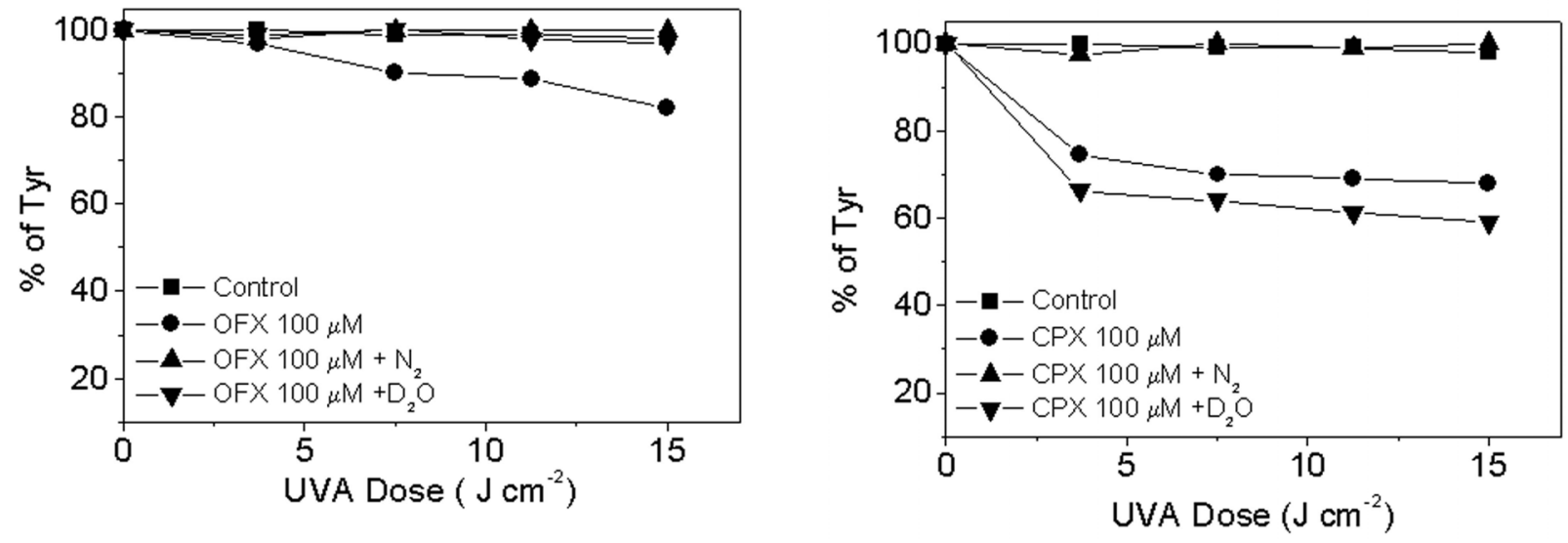

Figure 11. Photosensitising effects on Tyr by CPX, and OFX at the concentration of $100 \mu \mathrm{M}$ after irradiation in different conditions.

As can be observed from figure 11, CPX after irradiation reduces the percentage of Tyr in solution in agreement with the experiment carried out with RNAse. This effect is oxygen dependent as demonstrated by the protection afforded to Tyr when the experiments were conducted in anaerobic conditions. In deuterated buffer we observed, in the limits of experimental error, an effect not significantly different from that observed in aqueous buffer suggesting that among the different reactive oxygen species, ${ }^{1} \mathrm{O}_{2}$ does not play an important role. The effect of OFX was very weak and reached, at the highest UVA dose utilized, a reduction in Tyr content of about $20 \%$, while LMX and NOR do not induce any effects on this aminoacid.

Another amino acid studied was Histidine (His); photodegradation of His should be expected if the drug acts as a singlet oxygen sensitizer (type II mechanism). His is the aminoacid which reacts faster which singlet oxygen than other aminoacids such as $\operatorname{Trp}$ and $\mathrm{Tyr}^{22}$. As in the case of LMX, CPX and OFX (Figure 12) a weak decrease of His content, about $15 \%$, was observed upon irradiation in the presence of these drugs. This effect was eliminated in $\mathrm{N}_{2}$-purged solution but strongly potentiated in deuterated phosphate buffer indicating in this case the involvement of singlet oxygen. On the contrary, NOR has no effects in this test, even in the presence of deuterated buffer.
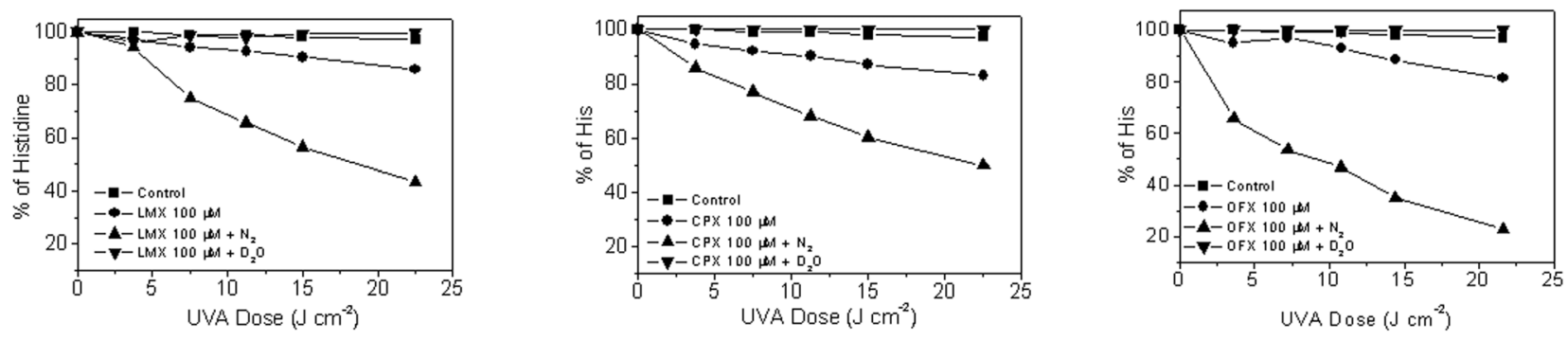

Figure 12. Photosensitising effects on His by CPX, LMX and OFX at the concentration of 100 $\mu \mathrm{M}$, after irradiation in different conditions. 


\section{Conclusions}

The results presented in this paper clearly demonstrated that fluoroquinolones are able to bind protein and upon UVA irradiation to induce various chemical changes in these macromolecules. In particular they are able to photooxidize proteins and to induce protein-protein cross-links both in isolated proteins (RNAse) or in erythrocyte membranes. In this case the formation of intermolecular cross-link induces an increase of the rigidity of the plasmatic cellular membranes with a consequent alteration of the cellular functions. It is known that protein cross-link occurs prevalently between His and Lys residues ${ }^{18}$ or with the formation of dityrosine bridges ${ }^{23}$. In this context we have demonstrated that the fluoroquinolones are able to photodamage some aminoacids in particular Tyr and His.

An important aspect to clarify with further experiments is the photohaptenic property of fluoroquinolones by which is originated their photoallergenicity ${ }^{21}$. The experiments carried out with LMX suggest the formation of a covalent adduct between the proteins and the drug.

In conclusion proteins can be considered a major target at molecular level in the mechanism of action of fluoroquinolones.

\section{Experimental Section}

Photophysical measurements. Absorption spectra were recorded with a Perkin-Elmer Lambda 800 spectrophotometer. Fluorescence spectra, corrected for the instrumental response were measured with a Fluorolog (Spex F112AI) spectrophotofluorometer (absorbance $<0.10$ at $\lambda_{\text {exc }}$ ). The transient behavior was investigated by use of a flash photolysis setup previously described $^{24,25}$ based on a Nd:YAG Continuum laser (Surelite II, third harmonics, $\lambda_{\text {exc }}=355 \mathrm{~nm}$, pulse width ca. $7 \mathrm{~ns}$ and energy $\leq 1 \mathrm{~mJ}$ pulse $^{-1}$ ). The transient spectra were obtained by monitoring the optical density change at intervals of 5-10 nm over the 300-800 nm range and averaging at least 10 decays at each wavelength. The kinetic analysis of the signals at selected wavelengths allowed the transient decay time to be determined; transient lifetimes were measured at an absorbance of ca. 0.2 ; the concentration effect on lifetimes was not investigated. All measurements were carried out at $22 \pm 2{ }^{\circ} \mathrm{C}$; the solutions were saturated by bubbling with argon. The experimental errors on $\tau_{\mathrm{T}}$ values were estimated to be about $\pm 10 \%$. All the measurements were carried out at low photon fluence and the samples were checked to avoid degradation.

Irradiation procedure. HPW 125 Philips lamps, mainly emitting at $365 \mathrm{~nm}$, were used for irradiation experiments. The spectral irradiance of the source was $4.0 \mathrm{~mW} \mathrm{~cm}$ as measured, at the sample level, by a Cole-Parmer Instrument Company radiometer (Niles, IL), equipped with a 365-CX sensor.

Erithrocyte ghost preparation. Ghosts were prepared from heparinized human blood following the gradual osmotic lysis method of Steck ${ }^{26}$. White membranes (ghosts) were resuspended in 
PBS buffer. Membrane protein contents were determined as described ${ }^{27}$, using BSA as a standard.

Protein photo cross-linking. The test compounds were added to the membrane suspension (1.0 $\mathrm{mg} / \mathrm{ml}$ protein concentration) and irradiated. The membrane samples were reduced and denatured by addition of $\beta$-mercaptoethanol and SDS at $90{ }^{\circ} \mathrm{C}$ for 3 minutes and bromophenol blue (BPB) was added before polyacrylamide gel electrophoresis analysis (5\% running gel, 3\% stacking gel). The quantitation of the bands, stained with Coomassie Brilliant Blue R-250 was achieved by image analyzer software Quantity One (BIO RAD, Milano Italy).

Analogous experiments were carried out in the presence of RNAse. Briefly protein solution $(0.5$ $\mathrm{mg} / \mathrm{ml}$ ) of RNAse in PBS buffer were irradiated for different times and analysed by SDS-PAGe gel electrophoresis (12\% running gel, $4 \%$ stacking gel).

Studies on isolated proteins. Solutions of Bovine serum albumin (BSA) $(0.5 \mathrm{mg} / \mathrm{ml})$ in phosphate buffer $10 \mathrm{mM}$ were irradiated in the presence of the test compounds for various times in a quartz cuvette. At each time the Tryptophan (Trp) content was followed by monitoring the characteristic Trp fluorescence as described by Balasubramanian et al., ${ }^{20}$. Solutions of Ribonuclease A (RNAseA), $0.5 \mathrm{mg} / \mathrm{ml}$ in phosphate buffer $10 \mathrm{mM}$ were irradiated in the presence of the test compounds for various times in a quartz cuvette. Analogous experiments were performed by bubbling nitrogen prior and during the irradiation.

The degree of protein oxidation was monitored spectrophotometrically, by the methods of Levine et al. ${ }^{19}$, by derivatization with 2,4-dinitrophenylhydrazine (DNPH).

Studies on isolated aminoacids. For these experiments the irradiation system and the buffer were the same as those used for the irradiation of BSA. Tryptophan solutions $(100 \mu \mathrm{M})$ were irradiated in the presence of the test compounds and analysed as described for BSA. Histidine solutions were irradiated for various time intervals and the resulting samples were analysed for His content as described by Figureido et al. ${ }^{28}$ Tyrosine solutions $(1 \mathrm{mM})$ were also irradiated in PBS and the resulting samples were analysed for Tyr content as described ${ }^{29}$. Analogous experiments were performed by bubbling with nitrogen before and during the irradiation and also using deuterated phosphate buffer.

\section{Acknowledgements}

The authors gratefully acknowledge the financial support of the Università di Perugia and Padova and of Ministero per l'Università e la Ricerca Scientifica e Tecnologica (Rome). 


\section{References}

1. Andriole, V. T. Drugs 1999, 58 (suppl.2), 1.

2. Domagala, J. M. J. Antimicrob. Chem. 1994685.

3. Stahlmann, R.; Lode H. In The quinolones; Andriole V. T. Ed... Academic Press: San Diego, 1998; pp 369-415.

4. Hooper, D. C.; Wolfson J. S. Antimicrob. Agents Chemother. 1985, 28, 716.

5. Wagai, N.; Tawara K. Arch. Toxicol., 1991, 65, 495.

6. Wagai, N.; Tawara K. Arch. Toxicol., 1992, 66, 497.

7. Ray, R. S.; Agrawal, N. ; Misra, R. B. ; Farooq, M. ; Hans, R. K. Drug Chem. Toxicol. 2006, $29,25$.

8. Umezawa, N. K.; Arakane, R. A. ; Mashiko, S.; Hirobe, M.; Nagano, T. Arch. Biochem. Biophys. 1997, 342, 275.

9. Martinez, L.; Chignell, C. F. J. Photochem. Photobiol. B: Biol. 1998 45, 51.

10. Martinez, L. J.; Sik, R. H.; Chignell, C. F. Photochem. Photobiol., 1998, 67, 399.

11. Wada, K.; Saniabadi, A. R.; Takiguchi, Y.; Nakashima, M. Pharm. Toxicol. 1994, 74, 240.

12. Schatchard, G. Ann. N.Y. Acad. Sci. 1949, 51, 660.

13. Kamat, B. P. J Pharm. Biom. Anal. 2005, 39, 1046.

14. Ahmad, B.; Parveen, S.; Khan, R. H. Biomacromol. 2006, 7, 1350.

15. Albini, A.; Monti, S. Chem. Soc. Rev. 2003, 32, 238.

16. Cuquerella, M. C.; Bosca, F.; Miranda, M. A. J. Org. Chem. 2004, 69, 7256.

17. Merville, M. P.; Piette, J.; Decuypier, J.; Calberg-Bacq, C. M.; Van De Vorst, A. Chem. Biol. Interactions, 1983, 44, 275.

18. Shen, H. R.; Spikes, J. D.; Kopockovà, P.; Kopecek, J. J. Photochem. Photobiol. B: Biology, 1996, 35, 213.

19. Levine, R. L; Garland, D.; Oliver, C. N.; Amici, A.; Climent, I.; Lenz, A. G.;. Ahn, B. W.; Shaltiel, S. W.; Stadtman, E. R. Methods Enzymol. 1990, 186, 464.

20. Balasubramanian, D.; Zigler, J. S. Photochem. Photobiol. 1990, 52, 761.

21. Tokura, Y.; Nishjima, T.; Yagi, H.; Furukawa, F.; Takigawa, M. Photochem. Photobiol. 1996, 64, 838.

22. Michaeli, A.; Feitelson, J. Photochem. Photobiol. 1994, 59, 284.

23. Kanwar, R.; Balasubramian, D. Biochemistry, 2000, 39, 14976.

24. Görner, H.; Elisei F.; Aloisi G. G. J. Chem. Soc., Faraday Trans. 1992, 88, 29.

25. Romani, A.; Elisei, F.; Masetti, F.; Favaro, G. J. Chem. Soc., Faraday Trans. 1992, 88, 2147.

26. Steck, T. L.; Kant, J. A. Methods Enzymol. 1974, 31, 172.

27. Peterson, G. L. Anal. Biochem. 1977, 83, 344.

28. Figureido, A.; Ribeido, C. A. F.; Goncolo M.; Baptista A. P.; Texeira, F. J. Photochem. Photobiol. B.; Biol. 1993, 18, 161.

29. Miranda M. A.; Castell J. V.; Hernandez, D.; Gomez-Lhecon, M. J.; Bosca, F.; Morera I. M.; Sarabia, Z. Chem. Res. Toxicol. 1998, 11, 172. 